\title{
A Meta-Analysis of Acupuncture for Chronic Pain
}

\author{
MAHESH PATEL, * FELIX GUTZWILLER, * FRED PACCAUd* AND ALFIO MARAZZI* *
}

Patel M (Route de Saint-Loup 4, 1290 Versoix, Switzerland), Gutzwiller F, Paccaud F and Marazzi A. A meta-analysis of acupuncture for chronic pain. International Journal of Epidemiology 1989, 18: 900-906.

Results of 14 randomized controlled trials of acupuncture for chronic pain were pooled in a meta-analysis and analysed in three subgroups according to site of pain; and in two subgroups each according to type to trial, type of treatment, type of control, 'blindness' of participating agents, trial size, and type of journal in which results were published. While few individual trials had statistically significant results, pooled results of many subgroups attained statistical significance in favour of acupuncture. Various potential sources of bias, including problems with blindness, precluded a conclusive finding although most results apparently favoured acupuncture.

While acupuncture is increasingly used by the general public and treatment costs are often reimbursed by health insurance companies, its clinical efficacy remains scientifically unproven. ${ }^{1.2}$ This study was undertaken to investigate the hypothesis that the individually inconclusive trials performed to date might, when their results were pooled in a meta-analysis (MA), yield a more definitive result.

\section{MATERIAL}

This MA is based on results of all trials of acupuncture for treatment of chronic pain, published in English, listed in Index Medicus from 1970 onwards that were randomized controlled trials (RCTs) of chronic pain that measured outcome in terms of number of patients whose condition improved. The World Health Organization's collection of (English language) Chinese, traditional, and alternative medical journals and literature yielded additional trials. References were obtained from previous reviews ${ }^{3.4}$ and from Catherine Hill's, as yet unpublished, excellent and comprehensive bibliography.

Trials were discarded if they were uncontrolled, ${ }^{3-10}$ not randomized ${ }^{11-13}$ or did not measure results in terms of numbers of patients improved and provide the number of patients randomized. ${ }^{14-16} \mathrm{~A}$ complete list of excluded trials is not presented.

Published study plans of the selected RCTs are summarized in Table 1. Few published baseline data after

\footnotetext{
-Route de Saint-Loup 4, 1290 Versoix, Switzerland.

**Institut Universitaire de Medecine Sociale et Preventive, 17 Rue du Bugnon, 1005 Lausanne, Switzerland.
}

randomization. ${ }^{17}$ Additional technical, ${ }^{18}$ methodological $^{19}$ and paradigmatic ${ }^{20}$ problems in evaluation of alternative medicine have been reviewed elsewhere.

Formula acupuncture (FA) uses a set of fixed points repeatedly. Classical acupuncturists (CA) traditionally vary points used from patient to patient, and from treatment to treatment. Most trials achieved 'Teh Chi' or a 'needling' feeling, ie numbness in the area of the needle, proof that a point has been located correctly.

The 'control' was sometimes a continuation of medical treatment. Although these RCTs focused on chronic rather than acute pain, thus reducing the probability of remission, use of continued conventional treatment controls is still unsatisfactory. Transcutaneous neural stimulation (TNS) was often used, sometimes on acupuncture points. Treatment of chronic pain with medical placebo (sugar pills) was not performed. Placebos most frequently used included placebo acupuncture and mock TNS. Clearly, blindness of patients was possible only when placebo acupuncture was used. Relative costs were not mentioned in any trial.

\section{METHOD}

'Meta-analysis"'.22 is a set of methodological techniques used to define accumulated knowledge by pooling results of studies. ${ }^{23}$ Methods used differ according to homogeneity of study outcome. RCTs selected were tested for homogeneity using the ' $Q$ ' statistic. ${ }^{24}$ The overall pooled risk difference and its $95 \%$ confidence interval $(\mathrm{Cl})$ between acupuncture and control groups was evaluated on the basis of a 'random effects' model, necessary as a result of the lack of underlying homog- 
TABLE 1 Acupuncture studies

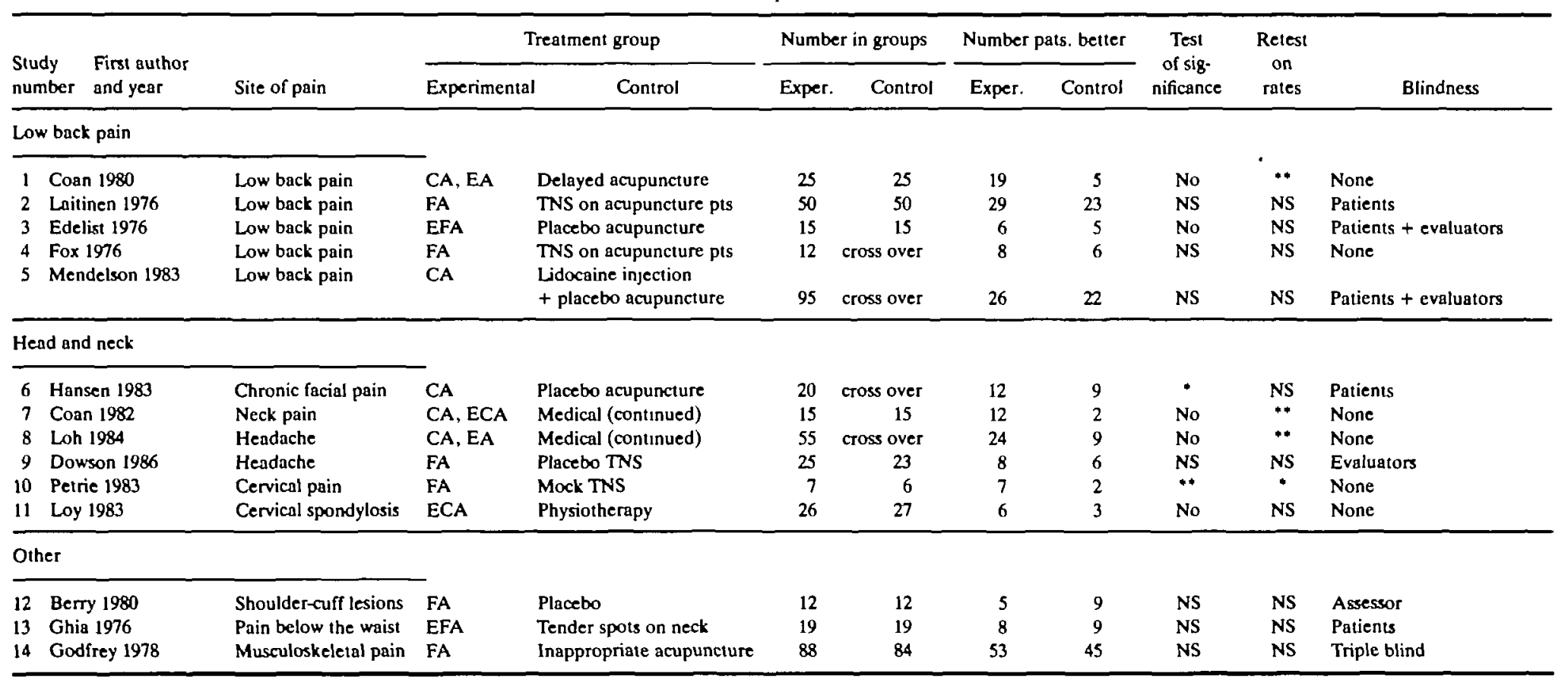

Note: Studies numbered as in Figure 1. Crossover studies summed over treatment order.

Legend: $C A=$ acupuncture point selection varied according to the needs of the patient.

FA = formula acupuncture, a standard set or sets of points applied to all patients.

ECA or EFA a acupuncture or formula acupuncture in conjunction with electrical stimulation of the needles.

TNS $=$ transcutaneous neural stimulation.

$=p<0.05 . \cdots=p<0.01$. NS $=$ no significant difference. 
eneity of the studies. Cochran's semi-weighted estimator for the risk difference was used. ${ }^{24}$ The results of two additional indicators, the logarithm of the odds ratio, and the logarithm of one minus (1-) the relative difference, were also examined. ${ }^{25}$

Complete information on crossover studies was unavailable in published trial reports, and these were treated as two independent samples summed over treatment order. If there is agreement between treatments, this procedure simply loses power. ${ }^{\text {.s }}$ In order to be certain that confusion was not generated by procedural differences between crossover and standard randomized trials, these were also analysed as two separate subgroups for each pain site.

For detailed evaluation, trials were classified (Table 2) into one of three subgroups according to the general anatomical site of pain (lower back, head and neck, and other sites) and into one of two subgroups according the nature of the control (placebo or treatment). Classical acupuncture was distinguished from formula acupuncture. Trials in which any agents (patients, therapists, or evaluators) were blind were identified. Large trials, 50 patients or more, were identified. Trials published in journals with the words 'Chinese' or 'acupuncture' in their title were distinguished from those in 'traditional western' medical journals.

Due to the large number of these classification criteria and the small total number of trials, subgroups based on combined classification criteria (eg. partially blind trials using classical acupuncture) were not exhaustively analysed. There are 192 ways in which

TABLE 2 Composition of subgroups

\begin{tabular}{ll}
\hline Subgroup (Homogeneity) & Study numbers' \\
\hline Low back pain** & $1,2,3,4,5$ \\
Head and neck pain** & $6,7,8,9,10,11$ \\
Other site of pain** & $12,13,14$ \\
Crossover design** & $4,5,6,8$ \\
Standard design** & $1,2,3,7,9,10,11,12,13,14$ \\
Placebo control** & $3,6,9,10,12,14$ \\
Conventional treatment** & $1,2,4,5,7,8,11,13$ \\
Classical acupuncture** & $1,5,6,7,8$ \\
Formula acupuncture* & $2,3,4,9,10,12,13,14$ \\
Some blindness* & $2,3,5,6,9,12,13,14$ \\
No blindness** & $1,4,7,8,10,11$ \\
Large trial (50 or over)*** & $1,2,5,8,11,14$ \\
Small trial (less than 50$)^{* *}$ & $3,4,6,7,9,10,12,13$ \\
Chinese medical joumal** & $1,2,7$ \\
Mainstream medical journal** & $3,4,5,6,8,9,10,11,12,13,14$ \\
\hline
\end{tabular}

'Studies numbered as in Table 1.

- Studies not homogeneous at $p<0.05$.

* Studies not homogeneous at $p<0.01$. two criteria could be combined. Hence residual, and potentially relevant, heterogeneity within initial groups could not be systematically avoided.

Editors may have been biased against publishing inconclusive studies. Pooling of published studies would then obtain a biased result. The influence of publication bias was evaluated by estimating the number of unpublished randomized controlled trials of acupuncture for the treatment of chronic pain with inconclusive results that would need to exist in order to negate the findings obtained ${ }^{26}$ (the 'file drawer' problem).

In order to ensure that MA methodology was comprehensively applied, the analysis was measured against a list of qualities and a scoring system proposed for medical MAs. ${ }^{27}$

\section{DATA}

Five trials dealing with low back pain (trials 1 to 5 in Table 1) met the selection criteria. Coan did not report statistical analysis of results. ${ }^{28}$ Results were statistically significant though long-term follow-up showed regression of beneficial effects. Laitinen ${ }^{29}$ and Edelist ${ }^{30}$ did not attain significance. Fox ${ }^{31}$ inserted only three needles, unilaterally, for one minute at each point sequentially. These third and fourth trials offered less treatment than is conventional. In Mendelson, ${ }^{32}$ both patients and the final evaluator of pain were blind, potentially confusing effects of treatment order were noted, and no statistically significant results were obtained.

The second group treated headache, neck pain, cervical pain, cervical spondylosis and chronic facial pain and included six trials that met the selection criteria (trials 6 to 11 in Table 1). In Hansen, placebo treatment involved superficial insertion of acupuncture needles at non-acupuncture points. A pain index yielded a Wilcoxson test with $0.05>p>0.025$, and a sign test of subjective preferences of patients yielded $p=0.035$, one-tailed, both in favour of acupuncture. ${ }^{33}$ $\mathrm{Coan}^{34}$ and $\mathrm{Loh}^{35}$ both attained statistical significance. These latter results in favour of acupuncture may have been pure placebo effects, as control groups merely continued medical treatment. Dowson ${ }^{36}$ used a true placebo (mock TNS), and did not obtain statistical significance. Cervical pain responded significantly $(p<0.01)$ to acupuncture in one small study ${ }^{37}$ but not in another. ${ }^{38}$

Three trials (trials 12 to 14 in Table 1) treated varied diagnoses. A single blind trial of multiple therapies for shoulder cuff lesion is one of two trials in which treatment did worse than control. ${ }^{39}$ The second evaluated acupuncture for chronic pain below the waist against 
'tender area needling'. Control did not explicitly exclude classical acupuncture points. ${ }^{40}$ In Godfrey 'most appropriate' acupuncture points, were compared to 'least appropriate' points. Triple-blindness may have been achieved. Directions were given by an acupuncturist who had evaluated the patient to another who could not see the patient (whose head was hidden by a screen). No statistically significant results were obtained. ${ }^{4}$

\section{RESULTS}

\section{Individual Trials}

A Chi-squared test on proportions of patients improved in studies that had not published statistical tests of results, ${ }^{28,34.35}$ yielded values of $p<0.01$. The risk difference is illustrated in Figure 1 for the 14 trials. Only two out of 14 trials obtained the result that patients treated with acupuncture did worse, on average, than the control group. The $95 \%$ CIs for four of these 14 trials did not include the 'zero risk difference' result. All four favoured acupuncture.

\section{Meta-analysis of Cumulated Trials}

Studies were not homogeneous according to the $Q$-statistic $(p<0.01)$. The overall risk difference (indicator ' $A$ ') between acupuncture and control groups was $0.184(\mathrm{SE}=0.062)$, in favour of acupuncture $(p<0.01)$. Acupuncture was also superior overall according to the logarithm of the odds ratio (indicator ' $B$ ') and the logarithm of ' $1 \longrightarrow$ the relative difference' (indicator ' $\mathrm{C}$ ').

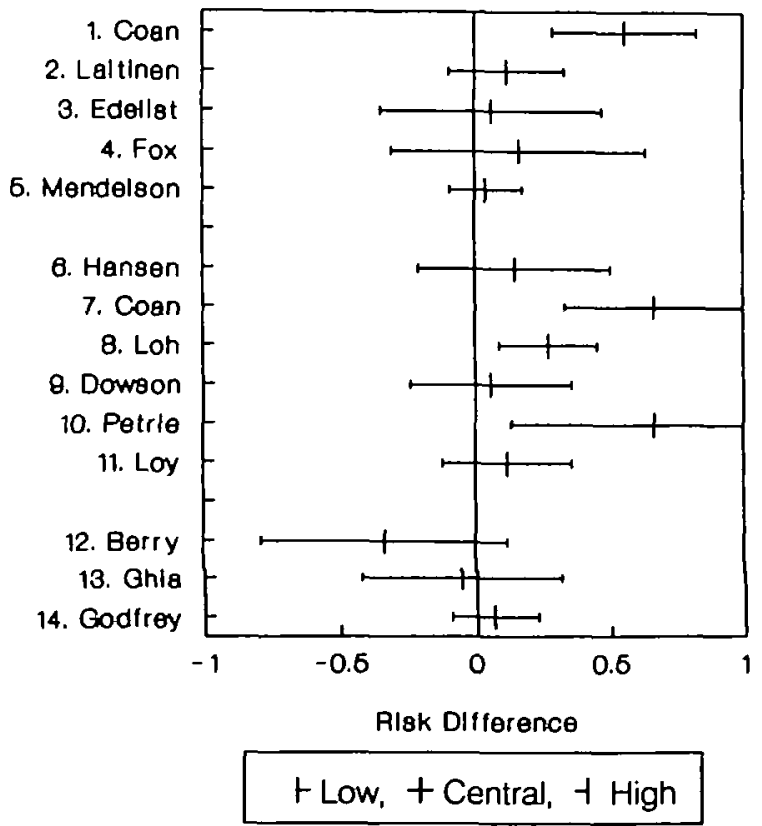

FIGURE 1. It RCTS of acupuncture; $95 \% \mathrm{Cl}$ for the risk difference.
None of the subgroups (Table 2) within which results were cumulated were homogeneous according to the ' $Q$ ' test ( $p<0.05$ for all subgroups).

Ninety-five per cent confidence intervals for the risk difference estimator (indicator ' $A$ ') are presented as Figure 2 . The results for all three indicators are presented as Table 3.

There was quite good agreement between results obtained from the three summary statistics used. In 14 of the 19 subgroups analysed, indicators ' $A$ ', 'B', and ' $\mathrm{C}$ ', provided the same result in terms of significance (or insignificance) of the difference between treatment and control groups.

Considering pooled results by site of pain, only the subgroup of trials for head and neck pain attained significance for all three indicators. Low back pain attained significance in favour of acupuncture according to indicators ' $B$ ' and ' $C$ ' if crossover trials were included. Results for other sites of pain showed an insignificant result in favour of the control group.

Acupuncture compared to conventional treatment was more favourable to acupuncture than trials against placebo. Patients receiving classical acupuncture at sites that varied from treatment to treatment did better than patients receiving formula acupuncture at fixed sites.

Trials with at least one blind agent were less favourable to acupuncture than trials without blind agents. Trials with some blindness did not attain significance for any indicator.

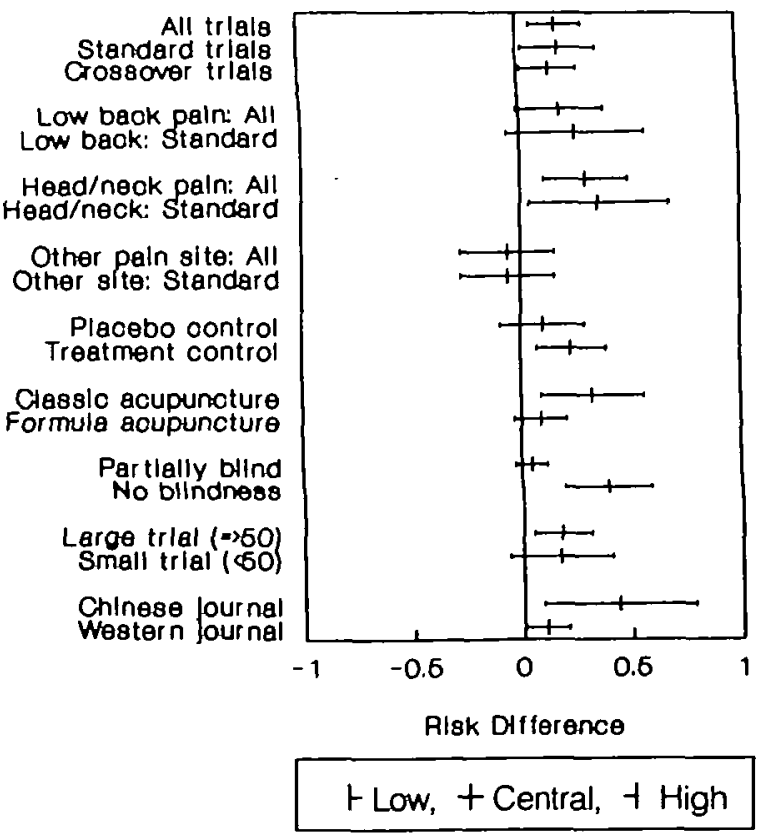

Figure 2. Pooled trial subgroups; 95\% CI for the risk difference. 
TABLE 3 Resuls obtained with meta-analysis of results of all trials and subgroups of trials defined, using the risk difference, the logarithm of the odds ration, and the logarithm of ' 1 -the relative risk difference', with tests of significance at $95 \%$ presented $(*)$

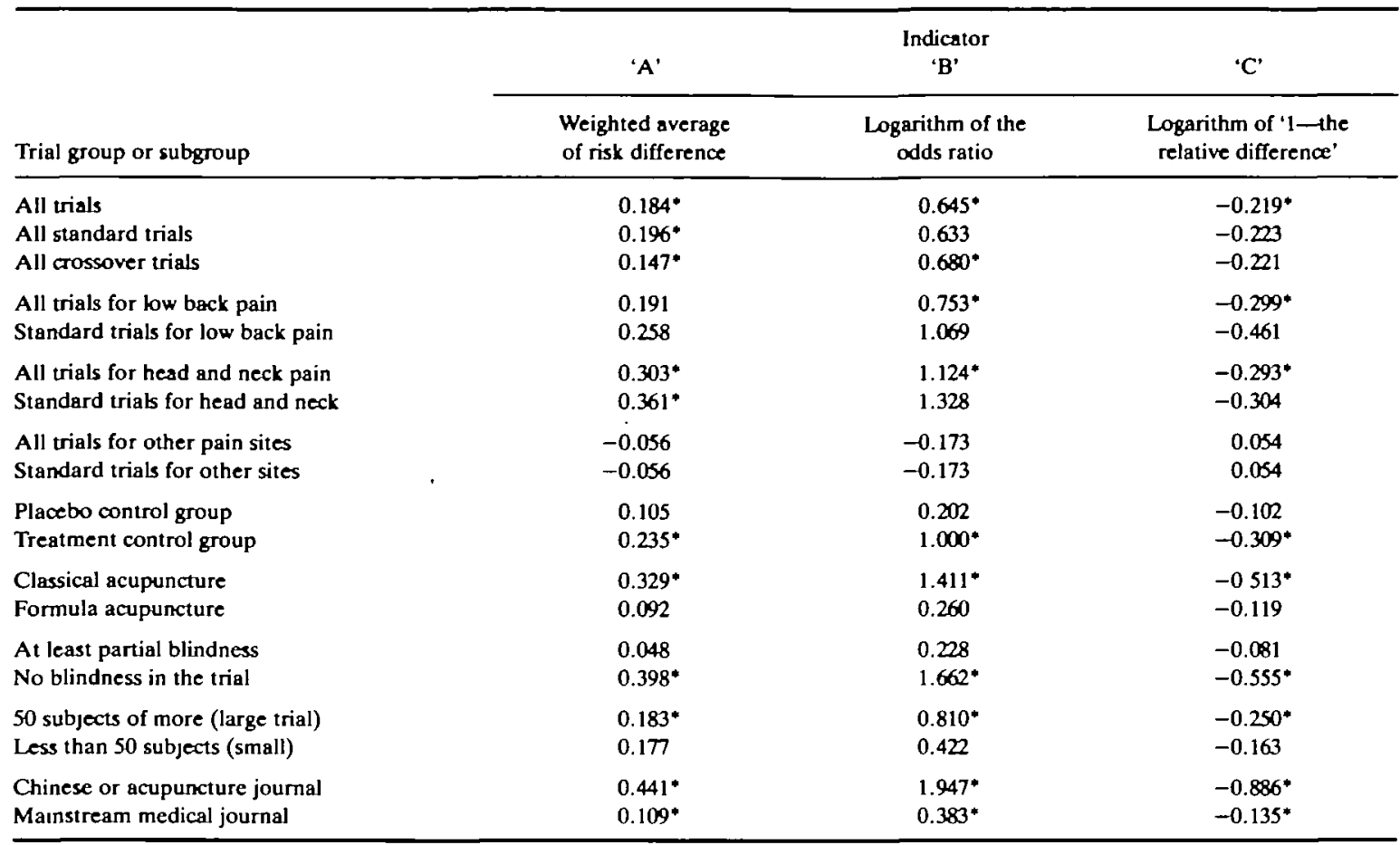

Large trials were more favourable to acupuncture than small trials. Results published in journals that had the word 'acupuncture' or 'Chinese' in their titles were significantly superior to those reported in 'traditional western' medical journals, but both these groups showed results favourable to acupuncture according to all three indicators.

The methodology of MA was comprehensively applied. Issues were covered in all six major areas of quality control of medical MAs. Twelve out of 23 relevant items were addressed. The analysis would be placed in the top decile of the 86 MAs reviewed by Sacks. That review obtained a mean of 7.7 items addressed with standard deviation of 2.7.

While some weaknesses of MA may not yet be fully appreciated, ${ }^{42}$ known problems certainly include 'publication bias' and 'author self-selection bias'. The necessary number of unpublished acupuncture RCTs for chronic pain that had, on average, inconclusive results (zero risk difference) to negate the statistical significance of the pooled result in favour of acupuncture was 26 trials at $p=0.01$, and 67 trials at $p=0.05$.

\section{DISCUSSION}

Blindness is the only means of avoiding bias due to preconceived notions of the superiority (or inferiority) of a treatment. The $95 \% \mathrm{CI}$ for the subgroup of 'trials with some blindness' includes the zero risk difference possibility for all indicators. If the four trials with statistically significant results are considered, only one common characteristic emerges. None had any degree of blindness. According to Godfrey, ${ }^{41}$ full triple blindness is technically feasible. If feasible, it should be regarded as essential.

There are two potential explanations for the apparent superiority of CA over FA. Either efficacy of CA is superior, or the protocols of CA trials were inferior. Two out of six CA trials, and six out of eight FA trials, displayed some degree of blindness.

The result that trials against conventional treatment are more favourable to acupuncture than are trials against placebo may similarly be explained. Five out of eight of the former had no blindness, and only one out of six of the latter.

The superior results obtained by trials published in journals oriented towards Chinese medicine or acupuncture may indicate selective publication bias. It is possible that acupuncture treatment described in specialist journals was superior, or that study methods differed between publication subgroups. All RCTs published in journals with the words China or acupuncture in their titles were trials against conventional treatment. 
It should be noted that, in MA, publication bias is made explicit. Individual studies that obtain positive results do not conventionally state that there may be large numbers of unpublished studies that obtained the opposite conclusion. The requirement that 67 inconclusive RCTs of acupuncture treatment for chronic pain exist to negate statistical significance of the pooled results is quite severe. No unpublished randomized controlled trials of acupuncture were discovered despite numerous contacts with the limited numbers of researchers in this area.

\section{CONCLUSIONS}

In pronouncing upon the efficacy, or otherwise, of a mode of treatment as contentious as acupuncture ${ }^{4-45}$ one is advisedly cautious in distinguishing between a statistically significant, and a conclusive, result. Results favourable to acupuncture were obtained significantly more often than chance alone would allow.

Publication bias may have influenced all the pooled risk difference estimates. As a result the true probabilities of type I and type II errors cannot be assessed. It is nevertheless considered 'very unlikely' that the 67 inconclusive RCTs required to negate the statistical significance of the pooled result exist.

Conclusive findings in favour of any new therapy can only be obtained from adequate triple blind randomized clinical trials. The published study plans of some trials depict a variety of deficiencies and stricter plans tended to yield less favourable results. The fact that results for acupuncture vary greatly according to the degree of blindness underlines this point.

Analysis of cumulated results of subgroups provides useful guidelines for future research. It is also possible that the choice between formula and classical acupuncture may influence results. Preliminary indications are that future trials should consider these as distinct types of treatment.

If acupuncture has a pain relieving effect, the mechanisms by which this effect could come about are, of course, unknown. However, while much more is known about the mode of function of effective analgesic drugs such as aspirin, the precise mechanism of this commonly used drug has not yet been completely understood. Acupuncture has probably been used more often than aspirin, worldwide. For all this experience, very little is known. Participation of academic medical departments and research institutions in systematic evaluation of acupuncture should therefore be encouraged to resolve these scientific issues.

\section{ACKNOWLEDGEMENTS}

We thank Monique Boehler and Catherine Hill for comments and invaluable help with documentation. Helpful comments were also received from Iain Chalmers, Guido Fisch, Bengt Jönsson, Harry Osore and Simon Page. No external funding was either requested or utilized in the course of this study.

\section{REFERENCES}

${ }^{1}$ Gutzwiller F, Chrzanowski R. Technology assessment: impact on medical practice. Int J Tech Assess Health Care 1986; 2: 99-106.

${ }^{2}$ Patel M S, St Leger A S, Schnerden H. Process and outcome in the National Health Service. Brit Med J 1985; 291 : 1365-6.

${ }^{3}$ Mendelson G. Acupuncture analgesia 1: Review of clinical studies. Aust NZ J Med 1977; 7: 642-8.

4 Richardson P H, Vincent C A. Acupuncture for the treatment of pain: A review of evaluative research. Pain 1986; 24: 15-40.

${ }^{5} \mathrm{Xu}$ Benren, Ge Shuhan. Observation of the effect of acupuncture treatment in 300 cases of primary trigeminal neuralgia. $J$ Trad Chin Med 1981; 1: 51-2.

${ }^{6}$ Laitinen J. Acupuncture for migraine prophylaxis: A prospective clinical study with six months follow-up. Am J Chin Med 1975; 3: $271-4$.

${ }^{7}$ Levine J D, Gormley J, Fields H L. Observations on the analgesic effects of needle puncture (acupuncture). Pain 1976; 2: $149-59$.

'Lee P K, Anderson T W, Modell J H, Saga S A. Treatment of chronic pain with acupuncture. JAMA 1975; 232: 1133-5.

' Borglum Jensen L, Tallgren A, Troest T, Börglum Jensen S. Effect of acupuncture on myogenic headache. Scand J Dent Res 1977; 85: $456-70$.

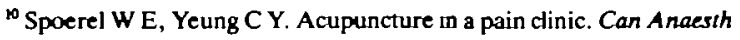
Soc J 1974; 21 : 221-9.

"Sodipo J O A, Falaiye J M. Acupuncture and gastric acid studies. Am J Chin Med 1979; 7: 356-61.

${ }^{1}$ Gunsberger M. Acupuncture in the treatment of sore throat symptomatology. Am J Chin Med 1973; 1: 337-40.

${ }^{13}$ Brattberg G. Acupuncture therapy for tennis elbow. Pain 1983; 16: $285-8$.

${ }^{14}$ Gaw A C, Chang L W, Shaw Lein-Chun. Efficacy of acupuncture of osteoarthritic pain: A controlled, double-blind study. $N$ Engl J Med 1975; 293: 375-8.

15 Johansson V, Kosic O, Lindahl L, Lindwall L, Tibbling L. Effect of acupuncture in tension headache and brainstem reflexes. $A d v$ Pain Res Ther 1986; 1: 839-41.

${ }^{16}$ MacDonald A J R, MacRae K D, Master B R, Rubin A P. Superficial acupuncture in the relief of chronic low back pain: $A$ placebo-controlled randomized trial. Ann $R$ Coll Surg Engl 1983; 65: 44-6.

${ }^{17}$ Ackermann-Liebrich U, Gutzwiller F, Keil U, Kunze M. Epidemiologie. Cham, Switzerland. Meducation Foundation.

1s Paccaud F. L'évaluation systématique des procédures médicales à propos de l'efficacité des "medecines paralleles". Rev Hed Suivse Romande 1986; 106: 141-7.

19 Patel M S. Evaluation of holistic medicine. Soc Sci Med 1987; 24: 169-75.

Datel M S. Problems in the evaluation of altemative medicine. Background paper, Section $L$ of the Xth International Conference of Social Science and Medicine. Soc Sei Med 1987; 25: 669-78.

${ }^{21}$ Patel M S. An introduction to meta-analysis. Health Policy 1989; 11 : $79-85$.

${ }^{2}$ Hunter J E, Schmidt F L, Jackson G B. Meta-analysis: Cumulating research findings across studies. Sage Publications, 1982.

$\checkmark$ The Lancet. Whither Meta-analysis? Lancet 1987; I: 897-8.

24 Der Simonian R, Laird N. Meta-analysis in clınical trials. Controlled Clin Trials 1986; 7: 177-88. 
Fleiss 3 L. Staristical methads for rates and proportions. John Wiley \& Sons, 1981.

${ }^{20}$ Rosenthal $R$. the "file drawer problem" and tolerance for null results. Psychol Bull 1979; 86: 638-41.

"Sacks H S, Berrier J, Reitman D, Ancona-Berk V A, Chalmers T C. Meta-analysis of randomized controlled trials. $N$ Eng $J$ Med 1987; 316: 450-5.

24 Coan $R$ M, Wong G, Su Liang Ku, Yick Chong Chang, Wang L, Ozer F, Coan P L. The acupuncture treatment of low back pain: A randomized controlled study. Am J Chin Med 1980; 8: $181-9$

Laitinen J. Acupuncture and transcutaneous electric stimulation in the treatment of chronic sacrolumbalgia and ischialgia. Am J Chin Med 1976 4: 169-75.

${ }^{-}$Edelist G, Gross A E, Langer F. Treatment of low back pain with acupuncture. Can Anaesth Sac J 1976; 23: 303-6.

${ }^{31}$ Fox E J, Melzack R. Transcutaneous electrical stimulation and acupuncture: Comparison of treatment for low-back pain. Pain 1976; 2: 141-8.

${ }^{7}$ Mendelson G, Selwood T S, Kranz H, Loh T M, Kidson M A, and Scott D S. Acupuncture treatment of low back pain: A doubleblind placebo-controlled trial. Am J Med 1983; 74: 49-55.

${ }^{3}$ Hansen P E, Hansen J H. Acupuncture treatment of chronic facial pain: A controlled crossover trial. Headache 1983; 23: 66-9.

${ }^{4}$ Coan $R$ M, Wong G, Coan P L. The acupuncture treatment of neck pain: A randomized controlled study Am J Chin Med 1982; 9: 326-32.
${ }^{3}$ Loh L, Natshan P W, Schott G D, Zilkha K J. Acupuncture versus medical treatment for migraine and muscle tension headaches. J Neurol Neurosurg Psychiarry 1984; 47: 333-7.

* Dowson D I, Lewith G T, Machin D. The effects of acupuncture versus placebo in the treatment of headache. Pain 1985; 21: $35-42$.

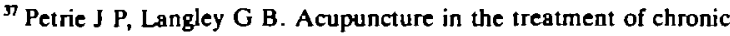
cervical pain: A pilot study. Clin Exp Rheumatol 1983; 1: 333-5.

- Loy T. Treatment of cervical spondylosis: Electroacupuncture versus physiotherapy. Med J A usi 1983; 3: 2-34.

"Berry H, Fernandes L, Bloom B, Clark R J, Hamilton E B D. Cinical study comparing acupuncture, physiotherapy, injection and oral anti-inflammatory therapy in shoulder cuff lessons. Curr Med Res Opin 1980; 7: 121-7.

- Ghia J N, Mao W, Toomey T C. Gregg J M. Acupuncture and chronic pain mechanisms. Pain 1976; 2: 285-99.

4" Godfrey C M, Morgan P. A controlied trial of the theory of acupuncture in musculoskeletal pain. $J$ Rheumatol 1978; 5: 121-4.

${ }^{2}$ Louis T A, Fineberg H V, Mosteller F. Findings for public health from meta-analyses. Ann Rev Public Health 1985; 6: 1-20.

13 Skrabanek P. Demarkation of the absurd. Lancet 1986; i: 960-1.

4 Fung K P, Lau S P. Acupuncture. Lancel 1984; I: $1169-71$.

4s Skrabanek P. Acupuncture and asthma. Letter. Lancet 1987; I: 1082-3.

(Revised version received April 1989) 